\title{
Contact Allergy in Atopic Patients: What We Suspected and What We Know
}

\author{
Andrea Nardelli, MD, $P h D^{1, *}$ \\ Carlos Consigli, MD, PhD \\ Premysl Bercik, $M D^{1}$ \\ Judy Wismer, $M D^{3}$
}

\author{
Address \\ ${ }^{*}{ }^{1}$ Farncombe Family Digestive Health Research Institute, McMaster University, \\ Hamilton, ON, Canada \\ Email: nardela@mcmaster.ca; andnardelli@hotmail.com \\ ${ }^{2}$ Department of Dermatology, Hospital Nacional de Clinicas, Universidad Nacional \\ de Cordoba, Cordoba, Argentina \\ ${ }^{3}$ Dermatology Division, Department of Medicine, McMaster University, Hamilton, \\ ON, Canada
}

Published online: 29 May 2015

(C) Springer International Publishing AG 2015

This article is part of the Topical Collection on Pediatric Allergy

Keywords Atopic dermatitis - Allergic contact dermatitis - Patch testing - Nickel - Fragrance - Sensitization - Chronic recurrent eczema . Irritants - Allergens . Pediatric - Piercing

\section{Opinion Statement}

Contact allergy in children was previously considered to be uncommon. However, data from the last decades have shown an increase in its prevalence, which is thought to result from more frequent exposure to allergens at a younger age, new trends in body piercing, use of cosmetic and pharmaceutical products, and participation in sports and hobbies. Factors that may influence the onset of sensitization in children are atopic dermatitis, skin barrier defects, and intense or repetitive contact with allergens. Although there are many similarities between adult and children with contact allergy, the patch test results obtained in adults cannot always be applied to children. Moreover, the notion of an association between atopy and contact allergy in children has been challenged. Experimental studies have shown that individuals with atopic dermatitis (AD) have suppressed contact sensitivity due to their disease. Patch testing should be considered at early stages in patients with $A D$; it is indicated not only when contact allergy is suspected but also in cases of persistent eczema on specific localizations, such as on the hands and the feet, around the mouth, and also in the umbilical region. Patients with AD should be tested when multiple exacerbations occur, even when they are treated, or when the dermatitis is asymmetrical. The most frequent contact allergens in children are metals, fragrances, hair dyes, adhesive and rubber chemicals, preservatives, and topical pharmaceutical products. 
Toys and portable electronic devices are another potential source of hapten exposure in children. Positive reactions in atopic children must be interpreted carefully, as atopic skin is readily irritated; this is specially the case for metals. Patch testing in children is safe, but false-positive reactions can occur. In addition, an abbreviated baseline series, supplemented with allergens suggested by the child's history, should be tested. The fundamental relationship between atopic disease and environmental chemical exposure in children is complex and occurs more tightly than previously supposed.

\section{Introduction}

Atopic dermatitis (AD) is a common skin condition characterized by dry and itchy skin with chronic or recurrent episodes of dermatitis that causes significant impairment in quality of life. Despite the fact that AD is mainly a disease of childhood, it may sometimes persist into adulthood and even may have a late onset [1]. The worldwide prevalence of $\mathrm{AD}$ has increased two- to threefolds over the 30 years and currently affects up to $20 \%$ of children and up to $5 \%$ of adults depending on the population $[2,3]$.

Although the pathogenesis of $\mathrm{AD}$ is complex, and many different pathways may be involved, it mainly results from disrupted barrier function and altered immune mechanism. As with other atopic disease, $\mathrm{AD}$ is often associated with the presence of protein sensitization (so-called extrinsic $A D$ ), characterized by positive tests [skin prick test (SPT) and/or radioallergosorbent test (RAST)] indicating IgE antibody to allergens, such as house dust mite, foods including egg and milk proteins, pollen, and animal dander [1]. A recent study has shown that intrinsic $\mathrm{AD}$, which is characterized by the absence of specific IgE to aeroallergens and food allergens, might be more associated with contact allergy to non-protein antigens [4], whereas extrinsic AD might be a form of systemic contact dermatitis to food and airborne protein allergens. Atopic patch testing (ATP) may be also performed as an additional test to diagnose delayed allergic outbreaks of $\mathrm{AD}$ to food and aeroallergen triggers of dermatitis in $\mathrm{AD}$ patients $[5,6]$.

The two major forms of contact dermatitis are irritant contact dermatitis (ICD) and allergic contact dermatitis (ACD). It may be difficult to distinguish between $\mathrm{AD}, \mathrm{ICD}$, and ACD because they all present as eczematous dermatitis and may coexist.
It is commonly accepted that $\mathrm{AD}$ patients may be more susceptible to ICD because of the compromised skin barrier in $\mathrm{AD}$ patients [7]. The presence of ICD in $\mathrm{AD}$ children is a common reason for seeking dermatology consultation.

Allergic contact dermatitis (ACD) is the most common occupational disease, in both children and adults, with prevalence reaching up to $20 \%$ [8]. ACD is a type IV delayed hypersensitivity reaction to allergens/haptens contacting the skin; it typically develops after repeated or prolonged topical exposure to chemical allergens of which are many hundreds.

Because $\mathrm{AD}$ is characterized by activation of inflammatory pathways and a disrupted epidermal barrier, increased permeation of allergens might lead to altered ACD reactions in a background of chronic AD [9]. The relationship between $\mathrm{AD}$ and ACD remains unclear, although AD is characterized by T-helper (Th) cell 2 phenotype in mainly the acute phase, with additional roles for Th1, Th17, and Th22 cells in chronic disease [10]. ACD is being increasingly recognized as a Th17- and Th22-polarized response in addition to a classic Th1 response [11]. Because of the relatively wide overlap of $\mathrm{AD}$ and $\mathrm{ACD}$, it is important to include patch testing in the diagnostic battery of physicians caring for children with AD.

The role of contact allergy in $\mathrm{AD}$ patients is frequently underestimated. Whether children with $\mathrm{AD}$ are more prone to $\mathrm{ACD}$ or/and have distinct contact hypersensitivity responses to common skin allergens than nonatopic children remains controversial. In this review article, we discuss factors that may influence this relationship, when and what allergens need to be patch tested and which are the most common allergens in $\mathrm{AD}$ children. 


\section{Prevalence of allergic contact dermatitis in atopic dermatitis children}

Sensitization to contact allergens can start in early infancy. The data on prevalence of contact allergy among children visiting dermatology clinics vary between 15 and $71 \%$ [12-15]. However, there are still few sufficiently large epidemiological studies on this subject [13-15]. The prevalence of contact allergy in children has increased over the last decades as a result of the more adult-like behavior of children, e.g., body piercing, hair dyeing, and use of cosmetics. A recent large study on ACD and children has shown that the sensitization rate was $25.1 \%$, and the prevalence of ACD was $16.7 \%$ [16•], these authors also shown that the likelihood of having at least one positive patch test reaction, mainly driven by nickel, and ACD was associated with the female gender. The overall impression was that contact allergy has become more frequent in the recent years, in particular from adolescence to adulthood, i.e., childhood atopic dermatitis and hand eczema were significant factors for hand eczema in adulthood when they were exposed to wet work [17•]. The rate of positive patch test results reflects not only different regional exposure patterns but also the local selection criteria and referral rules for patch testing and, finally, the compositions of local patch test series. Therefore, negative patch test results do not fully exclude ACD. False-negative reactions may, for instance, be due to a missing causative allergen, which could be identified by taking more detailed history. Moreover, misclassification of the clinical observation can occur, i.e., systemic contact dermatitis may appear like AD. This has been shown for several allergens that included nickel, chromium, and preservatives [18, 19]. Thus, there remains a knowledge gap regarding the exact prevalence of ACD in AD children.

\section{Factors that may influence the association between AD and contact sensitivity}

The epidermal barrier is crucial for the development of sensitization and elicitation of ACD. Recently, the key role of filaggrin (FLG) proteins in maintaining an effective skin barrier against the external environment has been demonstrated [20]. A positive association between FLG mutations and contact sensitivity (CS) to nickel was found among German adults [21], but could only be confirmed in Danish adults without ear piercings [22]. Furthermore, FLG mutation carriers reported ACD to nickel at a significantly younger age than controls with normal filaggrin, and they also displayed stronger patch test reactivity [23]. Nickel ions, like many other metal ions, are electrophilic in nature, which causes reactivity toward certain protein elements. It was hypothesized that those subjects who have FLG mutations are more susceptible to the acquisition of CS to nickel following topical exposure. This was supported by a study showing strong nickel chelation by the histidine-rich filaggrin proteins 
[24]. CS to metals has been found to have a significant association with $\mathrm{AD}$ in both children and adults, probably due to the compromised chelating func-

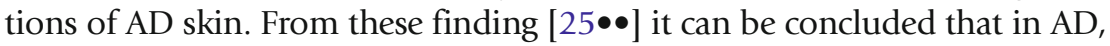
there may be an opportunity for more potent/easier access of chemical allergens to the viable epidermis, which could enhance the acquisition of CS. Moreover, another recent study in $\mathrm{AD}$ has shown that allergic immune reactions were globally attenuated and differentially polarized in patient with $\mathrm{AD}$, with significant decreases in Th1 products, some increases in Th17 activation, and inconsistent upregulation in levels of Th2 products. The overall skin hyporesponsiveness in patient with background $\mathrm{AD}$ might be explained by baseline immune abnormalities, such as increased $\mathrm{Th} 2, \mathrm{Th} 17$, and negative regulator levels compared with those seen in non-AD skin [26•].

The previous factors associated with $\mathrm{AD}$ and $\mathrm{CS}$ are more related to skin barrier status and immunological profiles of these entities. In the next section, environmental exposures and diagnostic factors will be discussed.

\section{Clinical pictures and patch testing in AD children}

It is of extreme importance to take a detailed history in children with dermatitis, in order to determine the environmental exposures of the children and those of their caretakers, and to examine thoroughly the topography of the lesions. It is particularly important to obtain a detailed history of the patients' exposure to hygiene products, clothing and footwear, jewelry, topical medications or herbal supplements, hobbies and extracurricular activities (athletic gear and equipment), recent travel, and environments. The localization is often an indication of the allergen or allergens involved. For example, one might suspect clothing allergens when the dermatitis is focused on those areas of the body such as the flexures of the extremities or subaxillary bands where garments may chronically rub against the skin. Facial, neck, or periorbital dermatitis may suggest allergy to preservatives or fragrances in cosmetic products [27]. A periumbilical, neck and wrist, or earlobe dermatitis might indicate exposure to nickel in the claps of jeans, necklace, bracelets, or earrings [28]. It is important to always ask about use of natural remedies in parallel to prescribed treatments, which may sustain the symptoms of ACD in children [29], with particular attention on the use of essential oil and Compositae dermatitis, which may appear as hand eczema in young children [30].

Sometimes the clinical picture is atypical. Certain substances are characteristic of children and may be responsible for an unusual clinical presentation $[31 \bullet \bullet]$. For example, tosylamide/formaldehyde in nail polish tends to cause ACD symptoms on the thinner skin of the eyelids and neck also called 'ectopic' dermatitis [27], as demonstrated in an atopic child in Fig. 1, but it spares the initial sites of contact on the thicker skin of her hands and fingers. In addition, many atopic children develop erythema and desquamation on plantar surfaces of both feet during spring or in relation to sports. This may lead to the misdiagnosis of shoes' contact allergy where potassium dichromate, rubber additive, p-pheny-lenediamine (PPD), and PTBF resin were described as the most common sensitizers [32]. Exposure to 'black henna' tattoos and to hair dyes are the main cause of strong patch test reactions to PPD in children aged 14 years and younger [33]. This has increased in the last years as temporary 


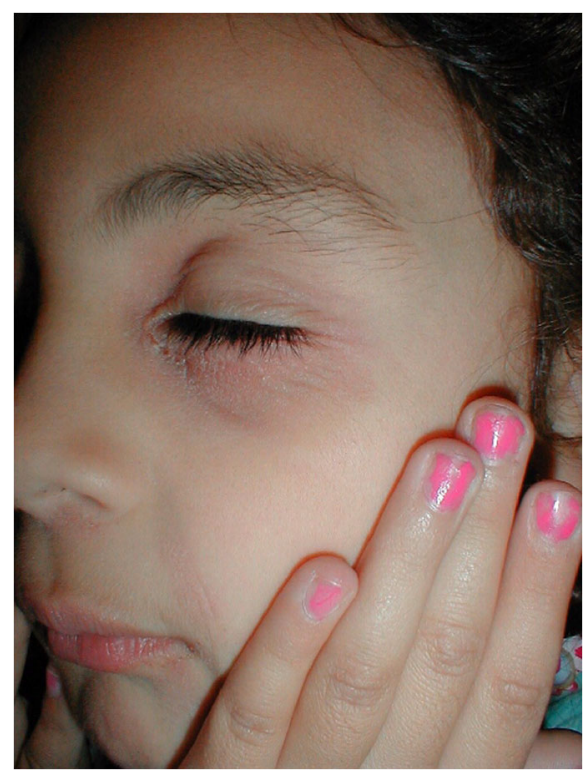

Fig. 1. A 6-year-old atopic girl with periorbital "ectopic dermatitis" due to tosylamide/formaldehyde in nail polish.

henna tattoos in children and early introduction of hair dyes became more widespread.

Parabens rarely cause sensitization on healthy, undamaged skin and are, therefore, considered safe in cosmetics. However, topical pharmaceutical preparations that are applied on damaged skin, particularly if water-based, may contain preservatives such as chlorocresol, phenoxyethanol, sorbic acid, or parabens. The diaper area is a particular environment where occlusion of the skin and urinary and fecal secretions lead to an increased humidity level and irritation, particularly in atopics, facilitating the penetration of potentially allergenic (and toxic) substances [34].

Methylisothiazolinone (MI) is frequently used in personal care products marketed for children, such as wet wipes, moisturizers, and body washes; this increased lately to epidemic proportions due to the massive increase in the use of cosmetic and other exposures [35]. Furthermore, oat and wheat have been described as contact allergens in personal care products, which can lead to protein contact dermatitis [36]. These aforementioned allergens may be the cause of flares in $\mathrm{AD}$ children.

Fragrance allergy in children seems to be common. This may be due to the promotion and use of perfumed products made specifically for children, including products for the diaper area and baby wipes, in which, beside fragrances also preservative agents as methyl(choro)isothiazolinone mixture are notorious sensitizers. Moreover, young children may 'play' with cosmetics, and girls are more likely to wear perfumed cosmetics and hair products, even those from their mothers [34]. The observation of simultaneous reactions to fragrance and Compositae plant extract (sesquiterpene lactones) can be explained by the common presence of terpenes [37]. For this reason, children with AD should be patch-tested with Compositae mix [37] relevant, for example, to feverfew in anti-itch medications. 
Portable electronic device are becoming increasingly popular among children of all ages. Significant nickel release has been associated with these devices. This nickel exposure can exacerbate $\mathrm{AD}$ and even result in generalized dermatitis [38-40].

Patch testing is indicated not only when contact allergy is suspected but also in cases of persistent eczema on specific localizations, such as on the hands and feet [41], around the mouth [28], and also in the umbilical region, particularly in AD children. The latter group should certainly be tested when multiple exacerbations occur, even when they are treated, or when the dermatitis is asymmetrical [42]. Occupational dermatitis, in particular, in adolescents with a positive history of AD and hand eczema should be considered since these conditions can be aggravated if the young adult decided to pursue a career in hairdressing, as a laboratory technician, dentistry/midwives, construction sector, as a metal worker, or in the food and plants industry. In all these professions, the risk of developing occupation dermatitis is increased [17].

Most authors agree that patch testing in children is safe [43], with problems being mainly technical due to the small patch test surface, inability to fix the patch test material to the skin due to patient's motion, which may result in loss of patch test materials, particularly in younger children, and the reluctance of some parents to allow patch testing. Table 1 shows practical instructions for testing children as adapted from Mallory [44].

Determining the relevance of the patch-test results can be difficult, especially in $\mathrm{AD}$ children. The underlying $\mathrm{AD}$ will not clear completely with avoidance of conventional contact allergens in contrast to non-AD children with purely ACD. Also, AD children who are tested during an active rash are at risk for false-positive angry-back reactions [45, 46]. In this respect, metals can provide a particular challenge due to changes in $\mathrm{pH}$ of the skin and nonspecific inflammation, resulting from penetration via sweat ducts and hair follicles [47]. Furthermore, irritant reactions to other chemicals are also common in $\mathrm{AD}$, the most important being to fragrances and formaldehyde in particular on early reading compared to controls [43].

Technical issues should be also considered in AD patients. Children often develop inflammation from occlusion such as that produced with

\section{Table 1. Practical instructions when testing children}

- Test in different sessions if the test area is very small.

- Should the patches come off, ask the parents to report it and instruct them not to reapply them.

- It may be necessary to use a stronger adhesive than usual, though this could result in local irritation.

- The application has to be performed as quickly as possible while the child is distracted.

- Make a diagram of the test allergens (this applies for adults, too).

- Inform the parents about the test procedure and the measures that may be taken to optimize the patch test conditions.

- Personal care products and medicament components in all patients should be tested.

- To avoid missing late reactions, it is recommended that patch test reading be carried out at D3/D4 and D5/D7.

- Test occupational contactants when indicated.

- Test foods in children with a history of perioral involvement. 
patch-test tapes [46]. Occlusion with synthetic fibers, such as polypropylene, has been shown to increase more staphylococcal proliferation in human sweat than occlusion with absorbent cellulosic fibers [48].

Staphylococcal colonization may proliferate under patch-test occlusion and cause flares, acting as either a superantigen or an allergen [49].

Bacterial colonization, e.g., colonization with gram-negative bacteria and Staphylococcus aureus is common in $\mathrm{AD}$ [1], likely increasing the risk of CS due to the presence of lipopolysaccharide that activates innate immunity via Toll-like receptors (TLR) and $S$. aureus exotoxins that target the $\mathrm{T}$ cell receptor (TCR) Vbeta 17 regions [50]. It is possible that bacterial colonization supports or enhances the acquisition of CS to at least some chemical allergens $[25 \bullet \bullet]$.

Patients with $\mathrm{AD}$ must be patch tested when the test site is not inflamed. Also, oral or intramuscular administration of corticosteroids should be avoided within 4 and 2 weeks, respectively, of the patch test or during testing as they extinguish the patients' response to the allergens. In addition, topical steroids should be discontinued to the back and upper arms, e.g., the sites of patch testing, 7 days before, and during the entire week of the patch test [46].

In view of smaller patch test area, especially with younger children, Brasch and Geier [43] proposed testing with an abbreviated baseline patch test series of 16 allergens. Roul et al. [32] also suggest reducing the number of tests: in children up to 6 years old, a series of 17 allergens; and in older, a baseline series of 29 to 31 allergens, depending on the testing center. Recently, Jacob et al. have suggested the use of at least the basic 20-allergen from North American pediatric series, in the routine screening of children ages 6-12 years [45]. These tests need to be completed with allergens depending on the clinical presentation and localization of the dermatitis. In cases where corticosteroids have been used, testing with corticosteroid allergy markers, tixocortol pivalate $0.1 \%$ and petrolatum and budesonide $0.1 \%$ (besides the corticosteroids used by the patient) is indicated [28].

Patch test relevance in children is best determined by observing complete resolution of dermatitis with the avoidance of identified allergens, but $\mathrm{AD}$ patients may also flare because of numerous triggering factors that are not identified by conventional patch testing. If this happens, it may be more difficult to determine the relevance of patchtest results [46].

Finally, meteorological and climate effects, such as a dry and arid climate resulting in xerotic skin tend to increase the rate of positive patch test reaction leading to false-positive results sometimes in children with AD.

\section{The relationship between atopic dermatitis and contact allergy. Unanswered questions}

It is understandable that neither experimental nor epidemiological studies can adjust for all the factors that may affect the association between AD and ACD in 
children. As we mentioned before, skin barrier dysfunction followed by increased bacterial colonization perpetuates the inflammation in AD. Several key questions regarding the mechanisms that drive the disease onset remain unanswered. These include:

1. Are changes in the microbiota composition the primary cause of the atopy or do they only reflect the enormous capacity of bacteria to adapt to new ecological conditions?

In most studies, the human microbiota associated with disease is characterized only after a disease phenotype has emerged, raising the important question of what came first: a disease or a change in the microbiome? Thus, it is important to characterize disease-associated changes in the microbiome before and after disease onset in $\mathrm{AD}$ children who develop ACD.

2. Unraveling principles and mechanisms that underline microbial community structure can help to understand the development of the atopic condition.

3. Does skin dysbiosis play a role in the pathophysiology of $\mathrm{AD}$ ?

4. Can therapeutic manipulation of skin microbial communities using pre- or probiotics, be helpful in maintaining human health? Perhaps the increased risk associated with the use of antibiotics is due to changes in the host microbiota, which lead to an altered development of the infant's immune system or an enhancement of the immune responses to environmental allergens

There is still insufficient information about long-term variations in the healthy/disease-associated microbiota in response to factors such as diet, environmental exposure, age, and therapeutic interventions to be able to draw robust conclusions regarding dysbiosis associated with disease [51]. Studies investigating the relationship between $\mathrm{AD}, \mathrm{ACD}$, and skin microbiota are warranted.

Contact allergy in children is more frequent than previously recognized. The history and localization of the dermatitis are crucial for the diagnosis of ACD, though certain allergens and/or habits that are characteristic for the child or the adolescent with $\mathrm{AD}$ may be responsible for unusual clinical presentations. Therefore, patch testing should be considered at an early point in children with a history of $\mathrm{AD}$ and active dermatitis, particularly if the distribution of the lesions is asymmetrical and persist when being treated. To avoid missing late reactions, it is recommended that patch test reading be carried out day 3, day 4, and days 5 and 7 .

It is still a matter of discussion whether ACD is more or less frequently associated with AD in children. Population-based and patientbased retrospective, epidemiological studies are needed to provide 
evidence for how contact sensitization affects the course of dermatitis in children with $\mathrm{AD}$. In the meantime, advice to parents should be based on the use of personal care products without fragrances and allergic preservatives in $\mathrm{AD}$ children. Parents and adolescent must be educated on alternative medicaments and personal care products in order to completely avoid identified allergens. Counseling is not only the most time-consuming but also the most critical aspect of treating AD. Finally, introducing new products on inflamed skin should be avoided as this may increase the risk of further sensitization.

\section{Compliance with Ethics Guidelines}

\section{Conflict of Interest}

Andrea Nardelli, Carlos Consigli, Premysl Bercik, and Judy Wismer declare no conflicts of interest.

Human and Animal Rights and Informed Consent

This article does not contain any studies with human or animal subjects performed by any of the authors.

\section{References and Recommended Reading}

Papers of particular interest, published recently, have been highlighted as:

- Of importance

-• Of major importance

1. Bieber T. Atopic dermatitis. N Engl J Med. 2008;358:1483-94.

2. Shaw TE, Currie GP, Koudelka CW, Simpson EL. Eczema prevalence in the United States: data from the 2003 National Survey of Children's Health. J Invest Dermatol. 2011;131:67-73.

3. Flohr C, Mann J. New insights into the epidemiology of childhood atopic dermatitis. Allergy. 2014;69:3-16.

4. Kabashima-Kubo R, Nakamura M, Sakabe J, et al. A group of atopic dermatitis without IgE elevation or barrier impairment shows a high Th1 frequency: possible immunological state of the intrinsic type. $\mathrm{J}$ Dermatol Sci. 2012;67:37-43.

5. Lipozencić J, Wolf R. The diagnostic value of atopy patch testing and prick testing in atopic dermatitis: facts and controversies. Clin Dermatol. 2010;28:38-44.

6. Darsow U, Laifaoui J, Kerschenlohr K, Wollenberg A, et al. The prevalence of positive reactions in the atopy patch test with aeroallergens and food allergens in subjects with atopic eczema: a European multicenter study. Allergy. 2004;59:1318-25.

7. de Jongh CM, Khrenova L, Verberk MM, et al. Loss-offunction polymorphisms in the filaggrin gene are

associated with an increased susceptibility to chronic irritant contact dermatitis: a case-control study. $\mathrm{Br} \mathrm{J}$ Dermatol. 2008;159:621-7.

8. Thyssen JP, Linneberg A, Menné T, Johansen JD. The epidemiology of contact allergy in the general population-prevalence and main findings. Contact Dermatitis. 2007;57:287-99.

9. Gittler JK, Krueger JG, Guttman-Yassky E. Atopic dermatitis results in intrinsic barrier and immune abnormalities: implications for contact dermatitis. J Allergy Clin Immunol. 2013;131:300-13.

10. Eyerich K, Novak N. Immunology of atopic eczema: overcoming the Th1/Th2 paradigm. Allergy. 2013;68:974-82.

11. Martin SF. Contact dermatitis: from pathomechanisms to immunotoxicology. Exp Dermatol. 2012;21:382-9.

12. Mortz CG, Andersen KE. Allergic contact dermatitis in children and adolescents. Contact Dermatitis. 1999;41:121-30.

13. Simonsen AB, Deleuran M, Johansen JD, Sommerlund $\mathrm{M}$. Contact allergy and allergic contact dermatitis in children-a review of current data. Contact Dermatitis. 2011;65:254-65. 
14. Jacob SE, Steele T, Brod B, Crawford GH. Dispelling the myths behind pediatric patch testing experience from our tertiary care patch testing centers. Pediatr Dermatol. 2008;25:296-300.

15. Zug KA, Pham AK, Belsito DV, et al. Patch testing in children from 2005 to 2012: results from the North American contact dermatitis group. Dermatitis. 2014;25:345-55.

16. Simonsen AB, Deleuran M, Mortz CG, et al. Allergic contact dermatitis in Danish children referred for patch testing-a nationwide multicentre study. Contact Dermatitis. 2014;70:104-11.

This is a very well design study on the long-term prevalence of ACD in Danish children.

17. Mortz CG, Bindslev-Jensen C, Andersen KE. Hand eczema in The Odense Adolescence Cohort Study on Atopic Diseases and Dermatitis (TOACS): prevalence, incidence and risk factors from adolescence to adulthood. Br J Dermatol. 2014;171:313-23.

This an elegant cohort study on the risk factors in adolescents that predict the development of an occupational contact dermatitis in the adulthood.

18. Shanon J, Pseudo-atopic dermatitis. Contact dermatitis due to chrome sensitivity simulating atopic dermatitis. Dermatologica. 1965;131:176-90.

19. Aerts O, Cattaert N, Lambert J, Goossens A. Airborne and systemic dermatitis, mimicking atopic dermatitis, caused by methylisothiazolinone in a young child. Contact Dermatitis. 2013;68:250-1.

20. Sandilands A, O'Regan GM, Liao H, et al. Prevalent and rare mutations in the gene encoding filaggrin cause ichthyosis vulgaris and predispose individuals to atopic dermatitis. J Invest

Dermatol. 2006;126:1770-5.

21. Novak N, Baurecht H, Schäfer T, et al. Loss-offunction mutations in the filaggrin gene and allergic contact sensitization to nickel. J Invest Dermatol. 2008;128:1430-5.

22. Thyssen JP, Carlsen BC, Menné T, et al. Filaggrin null mutations increase the risk and persistence of hand eczema in subjects with atopic dermatitis: results from a general population study. Br J Dermatol.

2010;163:115-20.

23. Ross-Hansen K, Menné T, Johansen JD. Nickel reactivity and filaggrin null mutations-evaluation of the filaggrin bypass theory in a general population. Contact Dermatitis. 2011;64:24-31.

24. Thyssen JP, Linneberg A, Ross-Hansen K, et al. Filaggrin mutations are strongly associated with contact sensitization in individuals with dermatitis. Contact Dermatitis. 2013;68:273-6.

25.• Thyssen JP, McFadden JP, Kimber I. The multiple factors affecting the association between atopic dermatitis and contact sensitization. Allergy. 2014;69:28-36.

This article is the most recent and thorough review on association between AD and ACD.

26. Correa da Rosa J, Malajian D, Shemer A, et al. Patients with atopic dermatitis have attenuated and distinct contact hypersensitivity responses to common allergens in skin. J Allergy Clin Immunol. 2015;135:712-20.

This article provides new insight in understanding the immunological mechanisms involved in $\mathrm{AD}$ and $\mathrm{ACD}$ and the overlap of these two conditions.

27. Militello G, Jacob SE, Crawford GH. Allergic contact dermatitis in children. Curr Opin Pediatr. 2006;18:385-90.

28. Goossen A, Morren M. In: Frosch PJ, Menné T, Lepoittevin JP, editors. Textbook of contact dermatitis. 4th ed. Berlin: Springer; 2006. p. 13-58.

29. Kütting B, Brehler R, Traupe H. Allergic contact dermatitis in children: strategies of prevention and risk management. Eur J Dermatol. 2004;14:80-5.

30. Paulsen E, Andersen KE. Sensitization patterns in Compositae-allergic patients with current or past atopic dermatitis. Contact Dermatitis. 2013;68:27785.

31.• de Waard-van der Spek FB, Andersen KE, Darsow U, et al. Allergic contact dermatitis in children: which factors are relevant? (review of the literature). Pediatr Allergy Immunol. 2013;24:321-9.

This article outlines the most common allergens in children and extensive description on the most characteristics clinical patterns of ACD that occur in children.

32. Roul S, Ducombs G, Taieb A. Usefulness of the European standard series for patch testing in children. A 3year single-centre study of 337 patients. Contact Dermatitis. 1999;40:232-5.

33. Spornraft-Ragaller P, Kämmerer E, Gillitzer C, Schmitt J. Severe allergic reactions to para-phenylenediamine in children and adolescents: should the patch test concentration of PPD be changed? J Dtsch Dermatol Ges. 2012;10:258-64.

34. Nardelli A, Morren MA, Goossens A. Contact allergy to fragrances and parabens in an atopic baby. Contact Dermatitis. 2009;60:107-9.

35. Aerts O, Baeck M, Constandt L, et al. The dramatic increase in the rate of methylisothiazolinone contact allergy in Belgium: a multicentre study. Contact Dermatitis. 2014;71:41-8.

36. Pootongkam S, Nedorost S. Oat and wheat as contact allergens in personal care products. Dermatitis. 2013;24:291-5.

37. Paulsen E, Otkjaer A, Andersen KE. Sesquiterpene lactone dermatitis in the young: is atopy a risk factor? Contact Dermatitis. 2008;59:1-6.

38. Jensen P, Johansen UB, Johansen JD, Thyssen JP. Nickel may be released from iPhone $\left({ }^{\circledR}\right) 5$. Contact Dermatitis. 2013;68:255-6.

39. Jensen P, Jellesen MS, Møller P, et al. Nickel may be released from laptop computers. Contact Dermatitis. 2012;67:384-5.

40. Jacob SE, Admani S. iPad-increasing nickel exposure in children. Pediatrics. 2014;134:580-2.

41. Isaksson M, Olhardt S, Rådehed J, Svensson Å. Children with Atopic Dermatitis Should Always be Patchtested if They Have Hand or Foot Dermatitis. Acta Derm Venereol. 2015;95:583-6. 
42. Shah M, Lewis FM, Gawkrodger DJ. Patch testing in children and adolescents: five years' experience and follow-up. J Am Acad Dermatol.

1997;37:964-8.

43. Brasch J, Geier J. Patch test results in schoolchildren. Results from the Information Network of Departments of Dermatology (IVDK) and the German Contact Dermatitis Research Group (DKG). Contact Dermatitis. 1997;37:286-93.

44. Mallory SB. The pediatric patient. In: Guin JD, editor. Practical contact dermatitis. New York: McGraw-Hill; 1997. p. 603-16.

45. Jacob SE, Admani S, Herro EM. Invited commentary: recommendation for a North American pediatric patch test series. Curr Allergy Asthma Rep. 2014;14:444.

46. Nedorost ST, Babineau D. Patch testing in atopic dermatitis. Dermatitis. 2010;21:251-4.
47. Storrs FJ, White Jr CR. False-positive "poral" cobalt patch test reactions reside in the eccrine acrosyringium. Cutis. 2000;65:49-53.

48. Teufel L, Pipal A, Schuster KC, Staudinger T, Redl B. Material-dependent growth of human skin bacteria on textiles investigated using challenge tests and DNA genotyping. J Appl Microbiol. 2010;108:450-61.

49. Werfel T. The role of leukocytes, keratinocytes, and allergen-specific IgE in the development of atopic dermatitis. J Invest Dermatol. 2009;129:1878-91.

50. Seo KS, Park JY, Terman DS, Bohach GA. A quantitative real time PCR method to analyze T cell receptor Vbeta subgroup expansion by staphylococcal superantigens. J Transl Med. 2010;8:2.

51. Nardelli A, Consigli C, Verdu EF, et al. Atopic dermatitis and skin microbiota. Contact Dermatitis. 2014;70 Suppl 1:53-72. 